\title{
A Web-Based Office Climate Control System Using Wireless Sensors
}

\author{
Timothy W. Foster, Deep Vardhan Bhatt, Gerhard P. Hancke, Fellow, IEEE, \\ and Bruno Silva, Student Member, IEEE
}

\begin{abstract}
Conventional heating, ventilating, and air conditioning (HVAC) systems usually achieve the desired control level by means of simple ON-OFF control, which can often result in high energy wastage. A potential solution to this issue is intelligent self-regulating HVAC controllers, which base their actions/decisions on sensor data. In this paper, an office climate monitoring and control system is designed and implemented. The system consists of various wireless sensor nodes and a control node. The sensor nodes provide the sensor data necessary to determine occupancy and the control node executes the algorithm, which decides whether to activate cooling or heating based on the sensor data. This system can serve as a controller and can be integrated into HVAC systems in smart buildings. It is shown that the developed control algorithm executed on the control node results in an improvement of up to $39 \%$ in energy efficiency over conventional ON-OFF controllers for HVAC systems.
\end{abstract}

Index Terms-Climate control, HVAC, thermal control, wireless sensor networks.

\section{Introduction}

I $\mathrm{N}$ RECENT times, there has been a growing demand for heating, ventilating and air conditioning (HVAC), especially from the perspective of smart and energy efficient buildings [1]. Advances in technology, as well as the widespread of ubiquitous wireless computing and Internet of Things (IoT), have consequently contributed to making these systems simpler and more cost effective [2]. Due to the widespread of such systems, it is estimated that $50 \%$ of energy in buildings is consumed by HVAC [1], [3]. In a lot of instances, this energy is generated using unclean fuels; therefore HVAC systems are usually responsible for a large global carbon footprint. A primary motivator for the development of a cost effective, smart HVAC system is the opportunity to greatly reduce energy consumption, and consequently the monthly operating cost of businesses employing HVAC. It is of utmost importance to have a HVAC system to ensure the occupants' comfort, especially in working environments. Previously developed HVAC systems have fallen short in a number of areas including energy efficiency, cost effectiveness and configuration complexity [1]. Traditional HVAC systems achieve the required control level by means of simple on-off control. These systems make use of an upper

The authors are with the Department of Electrical, Electronic and Computer Engineering, University of Pretoria, Pretoria 0002, South Africa (e-mail: u11044072@tuks.co.za; deep.bhatt@up.ac.za; gerhard.hancke@up.ac.za; silva.bjc@gmail.com)

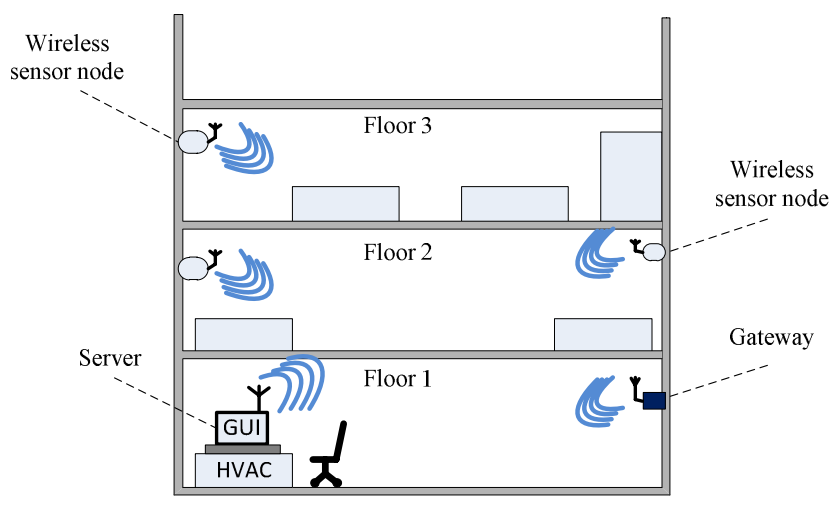

Fig. 1. Wireless sensor network for HVAC.

and lower temperature limit where the system is actively cooling the environment if the upper limit is reached, and heating the environment if the lower limit is reached. Better HVAC control can be achieved using intelligent occupancy detection, such that the system can autonomously adapt to the occupancy conditions. There are several commercial systems developed by companies such as Honeywell, Toshiba, Hitachi and Mitsubishi (amongst others) which offer a variety of sensors and controllers [4]-[6]. These systems are usually expensive and sometimes use proprietary wireless protocols and not popular wireless standards such as ZigBee (IEEE 802.15.4), which can be readily used in smart buildings [7]. Additionally, the majority of these controllers only have a local display that serves as the human machine interface (HMI) and do not offer the option to access/configure the controller remotely (eg. through a web interface) and they require on-off human control. A system which addresses these issues is desirable.

Advanced systems in this domain use proportional-integralderivative (PID) controllers (such as in the systems in [8]-[12]) which aim to minimize errors in the heating/cooling cycle, but sacrifice simplicity of installation and use. In order to compensate for varying load conditions, it is possible to implement self-tuning PID controllers [13]. This approach helps to improve performance, but still presents a challenge since it is difficult to obtain suitable models for HVAC systems. Thus, an intelligent controller which can self-optimize with the use of multiple sensors is potentially a viable solution to the problem of HVAC inefficiency. A wireless sensor system can be integrated into a building's HVAC [14], as illustrated in Fig. 1.

In this paper, we develop and implement such a system. This system considers sensor data (i.e. temperature, noise, 
light and occupancy) from various sensors placed at strategic locations within a room, and decides whether to activate cooling or heating based on the sensor data. The sensors include temperature and motion sensors (amongst others) which report their data in real-time to a control node responsible for executing a control algorithm to carry out the action.

Similar systems which also rely on wireless sensor networks include [15]-[17]. A comfort system based on IEEE 1451.2 is presented in [18]. It uses electrochemical and semiconductor sensors to sense temperature, humidity, carbon monoxide and carbon dioxide. However, the system has no interface to a mobile user and does consider motion, light nor noise, which are all parameters that can be used to increase the reliability of occupancy detection. Additional systems based on IEEE 1451.2 are also presented in [19] and [20]. In [6], a thermal comfort system aimed at HVAC applications was simulated but not implemented. In [21], three parameters were considered for comfort control. A volatile organic compound (VOC) wireless monitoring system based on ZigBee is presented in [22]. VOCs are emitted as gasses for certain liquids and solids (eg. paint strippers, pesticides, etc), and it is therefore crucial to monitor these gasses indoors for comfort.

The control system presented in this paper attempts to achieve greater energy efficiency than conventional HVAC systems by making use of wireless sensors. These sensor nodes monitor the environment and provide real-time data over a wireless connection to a control node, which then takes the appropriate action in quick response to changes in the environment. With regards to the wireless connectivity of the system, it is also important to ensure that enough high quality links are available to ensure good communication [23].

The system has been designed to reduce power consumption and wastage by increasing the efficiency of the HVAC system. This is achieved through the use of a network of wireless sensors which are used to sample the temperature (and other relevant parameters) at various points and determine occupancy of the room. Using this data, it is possible to improve the efficiency of standard HVAC systems by applying better control signals instead of simple on/off control. The primary focus of this work is on the implementation of the decision system used to select the appropriate action (i.e. lower or increase the temperature), and the sensor network which provides sensor data, and not on the implementation of a complete HVAC system. Furthermore, various sensors were tested to determine whether their data can be used to improve the occupancy detection rate. The goal is that the system developed here is able to interface with a HVAC system and server as a controller to said system. Integrating the system presented here into HVAC systems can result in greater HVAC efficiency, reduction of energy bills and reduction of carbon emissions. This paper makes the following contributions:

- the reliability of occupancy detection using different low cost sensors is evaluated;

- A controller algorithm which results in an energy efficiency improvement of 39\% over conventional on-off schemes to control HVAC systems is presented.
The rest of this paper is organized as follows: Section II gives discusses background work on occupancy detection; Section III provides a general overview of the system designed here; Section IV discusses the controller algorithm; Section V builds on Section III, and presents the detailed design and implementation of each sub-system; Section VI discusses the experimental protocol and results; and in Section VII the paper is finally concluded.

\section{Background:Occupancy Detection}

Occupancy detection is the action of determining if there are people present in locations such rooms or corridors. Examples include automatic lights, for instance. Using an occupancy sensor, it can be determined if there are people in the room, and lights can be switched on or off accordingly. Although various sensors exist and there is significant research on the topic, accurate occupancy detection is still very complex [24], [25]. Occupancy detection is closely intertwined with intelligent HVAC controllers, as the decisions made by the controller are based on the data provided by the occupancy sensors. In this section we discuss occupancy detection and give some background to place the system presented in this work in context.

\section{A. Passive Infrared Sensors, Ultrasonic Sensors and People Counting}

Passive infrared (PIR) sensors detect infrared radiation caused by the movement of the human body. It is a very popular type of sensor which is used in a lot of occupancy detection systems. A key limitation of PIR sensors is that they do not provide any information regarding the number of people in a room; they are only able to detect movement (i.e. whether a person has passed through the area covered by the sensor), and therefore they are rarely deployed without other sensors. Nevertheless, recent research has attempted to determine the number of people in a room using distributed PIR sensors [26]. Ultrasonic sensors operate based on the principle of Doppler shift, and similar to PIRs, are also used to detect presence. This type of sensor emits high frequency sounds which are then reflected by nearby objects. In the presence of a moving human being, the reflected pattern differs therefore it can be inferred that there's an occupant.

Alternative methods to get an estimate of the number people in a room (also known as people counting) at any given time are necessary. Promising approaches currently include camera based approaches [27], where is usually easier to perform (in comparison other types of sensors) because of the ability to actually view people. Ultra-wideband radars have also been proposed as a potential solution to the people counting problem [28]. Although the above-mentioned approaches have been proposed, it is still a real challenge to count people in a room accurately.

\section{B. Ambient Noise}

Noise has also been used (albeit to a less extent compared to the other sensing methods highlighted above) in 


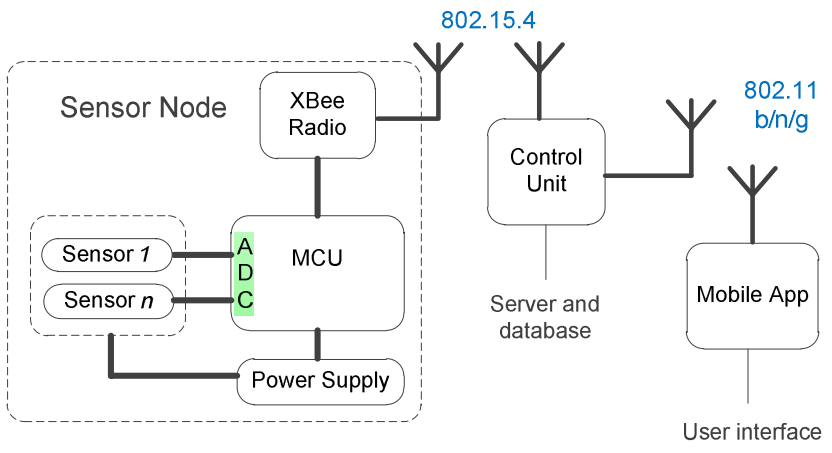

Fig. 2. Overview of the developed system.

occupancy detection. Acoustic sensing can be performed using microphones. By interpreting/classifying different kinds of sounds, it is possible to infer occupancy based on ambient noise by considering only specific sounds which are relevant for occupancy detection, such voices and sounds of people walking [29].

\section{Device-Free Localization}

Another popular technique that has emerged in recent years is device free (or passive) localization. Unlike active localization where sensors are attached to the objects/people to be tracked, device-free localization infers the presence of a human being by observing changes induced by moving objects on certain characteristics of wireless signals [30]. This can be directly applied to occupancy detection. Additionally, the deployed Wi-Fi infrastructure readily available in most buildings can be used for this purpose, which eliminates the need for additional dedicated infrastructure, unlike the other methods which have been discussed above. Recent research work which has investigated this includes [31].

\section{Algorithms for Occupancy Detection}

Once data has been collected from various sensors, algorithms are necessary to extract knowledge from the data to provide a reliable occupancy detection estimate. Techniques include artificial neural networks (ANN), support vector machines (SVMs) and hidden Markov models (HMMs). These have been applied in [32] and [33]. The features extracted from the data sourced from different sensors are used by these algorithms to detect occupancy. Examples of additional algorithms are discussed in [34] and [35]. In [36], an Autoregressive Hidden Markov Model (ARHMM) was developed to model the occupancy pattern using data from PIR sensors, carbon dioxide, concentration and relative humidity sensors. It was found that the algorithm has an average estimation accuracy of $80.78 \%$ and outperforms the other previously mentioned methods. A method based on electricity data consumption with an occupancy detection rate of $94 \%$ is presented in [37].

\section{Overview of the system}

The system consists of 3 main functional units (illustrated in Fig. 2): wireless sensor nodes, control unit and
Table 1: Wireless Sensor Node Hardware Details

\begin{tabular}{ll}
\hline \multicolumn{1}{c}{ Device } & \multicolumn{1}{c}{ Specification } \\
\hline Microcontroller & ATmega328P \\
Smoke sensor & MQ-2 \\
Noise sensor & CMA-4544PF-W \\
Temperature sensor & DS18B20 \\
Light sensor & CDS003 \\
Motion sensor & Ultrasonic range finders \\
ZigBee module & XB24-Z77CIT-004 \\
Wi-Fi module & Espressif ESP8266 \\
\hline
\end{tabular}

mobile application. The sensor nodes detect temperature, background noise, available light, occupancy and smoke.

For temperature, a DS18B20 one-wire digital sensor is used.

For occupancy detection, multiple ultrasonic range finders modules were placed at each of the entrances of a room and directed at the entrance, such that it is possible to detect whether a person is entering or exiting the room.

A CDS003 light dependent cell was used to estimate the lux, where an illuminance of 500 lux is typically recommended for normal office working areas. All sensor data is collected and transmitted to the control unit in a periodic fashion. To improve the energy usage of the system, temperature in a room is only monitored if it is known that the room is not empty. Noise level measurements were recorded with a CMA-4544PF-W electret microphone. An MQ-2 electromechanical gas sensor was used to detect the presence of smoke. This sensor can detect liquefied petroleum gas (LPG), propane, methane and alcohol. Table I summarizes the sensor system's specs.

Wireless communication between wireless sensor nodes and control node is performed using 802.15.4, and 802.11 is used between the mobile client and control mode through a wireless router or access point which is part of the wireless local network in the building. The control unit must be able to accurately make a decision on how to adjust the temperature of the environment by taking into consideration all data from the sensors. Each of these sub-systems is explained in more detail in Section V.

\section{Controller Algorithm}

This section demonstrates - through simulation - that a more advanced control algorithm outperforms the traditional on/off controlled systems. The algorithm discussed here is then implemented on the control node's microcontroller.

\section{A. Simulation Options}

HVAC has a dominant role in the overall energy efficiency of a building. By analyzing actual energy consumed versus the energy required for heating/cooling, it has been shown that the efficiency of HVAC systems can be improved on average by $30 \%$ [38]. In order to design the proposed system, it is crucial to understand how thermal energy behaves inside a building. For this purpose, energy 


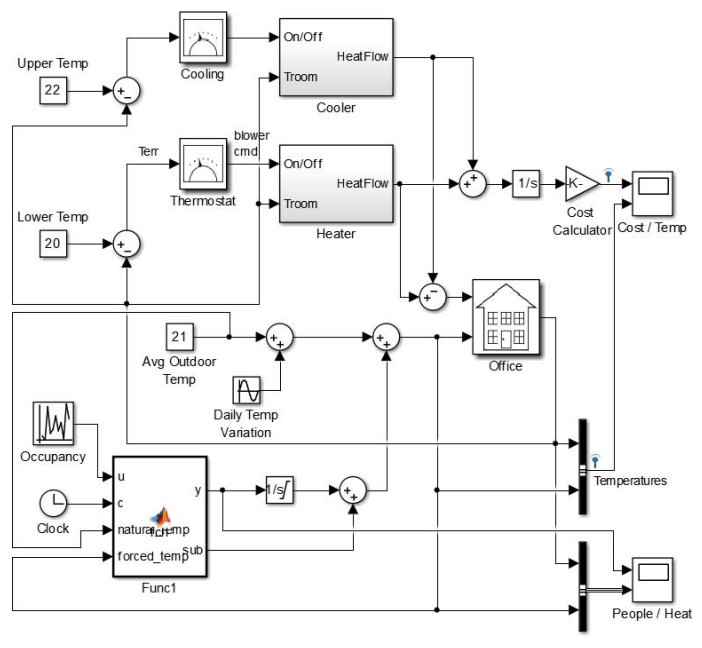

Fig. 3. Simulink model.
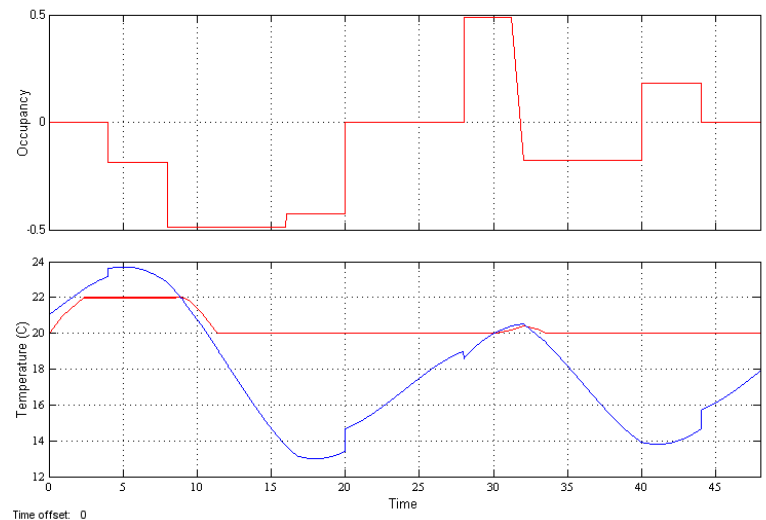

Fig. 4. Typical results produced by the Simulink model.

simulations which take into account several environmental variables (including occupancy, speed of airflow, etc), can be conducted [39] but often require complex mathematical models. Some simulation software such as DOE-2 [40] and TRNSYS [41], [42] can be used, but TRNSYS is not applicable for occupancy based design. Occupancy and other factors can be used to simulate and test alternative control algorithms [43]-[45]. Simulink is another popular option to simulate control systems.

\section{B. Simulation}

To show that on-off type control can be improved upon, a simulation on Simulink was conducted. This simulation was based on a simple thermodynamic model of an office floor and application of on-off functions to the heating and cooling functions. The Simulink model is illustrated in Fig. 3.

The energy cost was calculated by integrating the total energy output of the heating and cooling blocks and multiplying by a value of 0.9 cents per $\mathrm{kWh}$. Occupancy of the environment was generated and changed randomly throughout a 24-hour period (except for the hours between 20:00 and 6:00) wherein occupancy was reduced to zero to simulate a typical working day at the office. Fig. 4 shows occupancy on the top half and temperature at the bottom, and is a typical example

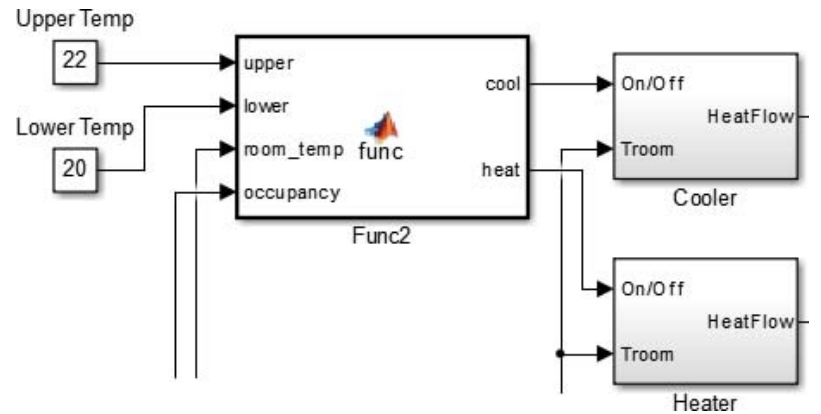

Fig. 5. Improved controller on Simulink.

of simulating the system over 48 hours. The occupancy value fluctuates between -0.5 and 0.5 , where this value represents the amount of heat given off by 10 people per hour, and was calculated by assuming that on average a person consumes $8.37 \times 10^{6}$ joules of energy (2000 calories) per day, and that this energy must be dissipated in the form of heat over the course of each day.

The occupancy value is then a measure of the total heat being added to the system by the room's occupants. Since this value is integrated to produce an actual temperature to add to the environment, the negative occupancy values indicate that someone has left the room. Thus, their effect on the temperature of the environment is gradually reduced over time.

Within the office model, the temperature of the room is calculated by subtracting the thermal losses of the building from the mass of air in the room, and then adding to this the heat flow of the cooler or heater, divided by the heat capacity of the air in the room.

The bottom half of Fig. 4 shows the on-off controlled HVAC system attempting to keep the temperature of the office between $20^{\circ} \mathrm{C}$ and $22^{\circ} \mathrm{C}$, in spite of the fluctuating temperature changes due to the behaviour of the people and the variations in outside temperature. This model was used to establish a baseline performance value in terms of cost, which was then compared with the performance of the improved system. The improved system was set up using a slightly modified version of the above model illustrated in Fig. 5 (sections which are exactly the same in Fig. 3 have been omitted for brevity).

In this simulation model, the improved algorithm was implemented in the block labelled "Func2" and the output of this function was used to trigger the heating and cooling in a similar fashion to the on-off controlled system. The algorithm that was implemented attempted to achieve better efficiency by regulating heating and cooling based on both occupancy and temperature. Firstly, minimum and maximum bounds were set to $2^{\circ} \mathrm{C}$ above the higher threshold and $2^{\circ} \mathrm{C}$ below the lower threshold, respectively. These values are considered to be within the human comfort zone when the humidity is between $46 \%$ and $56 \%$ for a set point of $21^{\circ} \mathrm{C}$.

Using the value for occupancy, the system either shuts down if occupancy is registered as zero or begins heating or cooling depending on the temperature or if occupancy is detected to be greater than two people. Finally, if occupancy is detected to be less than two people but the maximum or minimum bounds have been exceeded, the system activates. This setup 
successfully maintains human comfort levels whilst allowing the office temperature to drift towards the outside temperature. The flow diagram of the algorithm (as implemented on a microcontroller) described here is shown in Fig. 10.

\section{Sensor and Control Nodes: Deand Implementation}

This section describes the design and implementation of the sensor nodes and control node in more detail. This includes (i) development of a mobile application, (ii) communication between the control node and the local network over 802.11, (iii) communication between the sensor nodes and control node using 802.15.4, and (iv) sensing the environment.

\section{A. Microcontroller \& Wireless Connectivity}

The microcontroller selected for this system is an 8-bit ATmega328P microcontroller (MCU) with sufficient capabilities to meet the system's requirements. One of the requirements for the MCU was an analog-to-digital converter (ADC) module with at least a 10-bit resolution and 5 channels. All sensors used are connected to the MCU via ADC and the control algorithm is programmed onto the MCU. XBee XB24-Z77CIT-004 radios operating at a frequency of $2.4 \mathrm{GHz}$ were connected to each wireless sensor node and the control node.

\section{B. Web Server}

One of the requirements of the web server was to be able to handle at least 3 requesting clients simultaneously. To this end, a multithreaded webserver was implemented on an Espressif ESP8266 module. The server is able to handle incoming requests and serve the appropriate files, and it is also able to handle AJAX requests. However, due to memory limitations (instruction RAM of $64 \mathrm{KiB}$ and $96 \mathrm{KiB}$ data RAM) and processing speed, the module can run out of memory, execute kernel panic and restart. To avoid this, the web application and mobile application are designed to request information at $30 \mathrm{~s}$ intervals instead. The webpage was written using a combination of HTML5, CSS3, jQuery and the chart.js library.

\section{Noise Sensor}

The noise sensor is an electret microphone that is used to measure the ambient noise. It is a CMA-4544PF-W microphone which is omnidirectional and sensitive to within $+/-2 \mathrm{~dB}$ over the $20 \mathrm{~Hz}-20 \mathrm{kHz}$ frequency range. A MAX9814 amplifier connected to the microphone was configured to a gain of $40 \mathrm{~dB}$. The output of this sensor was then converted to an appropriate estimation of the noise in the environment by the software running on the sensor node's MCU.

\section{Motion Sensing}

In addition to the temperature in an office, another important factor is occupancy. Knowing the number of people in the

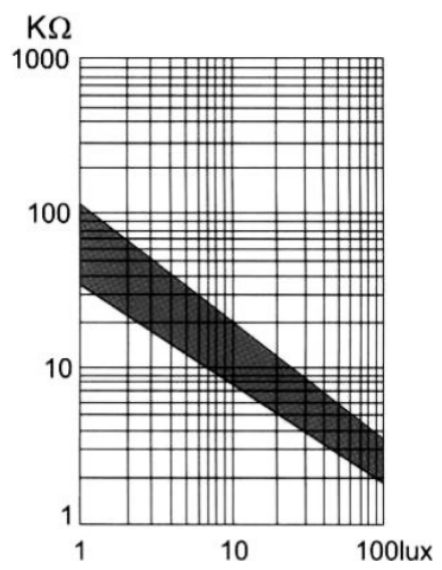

Fig. 6. Illuminance vs photo resistance.

room allows better control over the HVAC system. However, accurate determination of occupancy is challenging due to random human behavior. Occupancy in the proposed system was achieved using multiple sonic rangefinder modules located at each of the entrances to the room. These sensors were directed at the entrances, such that it is possible to detect if a person is entering (or exiting), based on the gradient of the readings obtained from the module. If a person is entering a room, the module shows readings which are successively decreasing. When a person is exiting the room, successive readings increase in value. Thus, it is possible to count the entry and exit of people at each entrance of the room. The control node then collates this information into an occupancy reading for the room.

An alternative would be to use light dependent resistors (LDRs) and corresponding laser diodes at each entrance, but this would require a lengthy installation procedure and significant amounts of wire.

\section{E. Light Sensor}

The light sensor was calibrated to operate within a range of 100 to 2000 lux. This range covers the majority of offices while still meeting the required specifications for accuracy. A CDS003 light dependent cell was connected in series to a $10 \mathrm{k} \Omega$ resistor, where the ADC of the microcontroller measured the center point. The information illustrated in Fig. 6 was taken from the cell's datasheet and subsequently used to calculate lux as follows.

From Fig. 6:

$$
\begin{aligned}
& P_{1}(\log 1, \log 100)=P_{1}(0,2) \\
& P_{2}(\log 10, \log 18)=P_{2}(1,1.255) \\
& P_{3}(\log 100, \log 3)=P_{3}(2,0.477)
\end{aligned}
$$

Using $P_{1}(0,2), P_{2}(1,1.255)$ and $P_{3}(2,0.477)$, an expression of the linear relationship can be obtained with $c=2.0165$ and $m=-0.7615$. This expression becomes:

$$
\begin{aligned}
\log (\text { lux }) & =-\frac{1}{0.7615} \log (R[k \Omega])+\log \left(10^{2.6726}\right) \\
& =\log \left(R[k \Omega]^{-1.3132}\right)+\log (470.5437)
\end{aligned}
$$




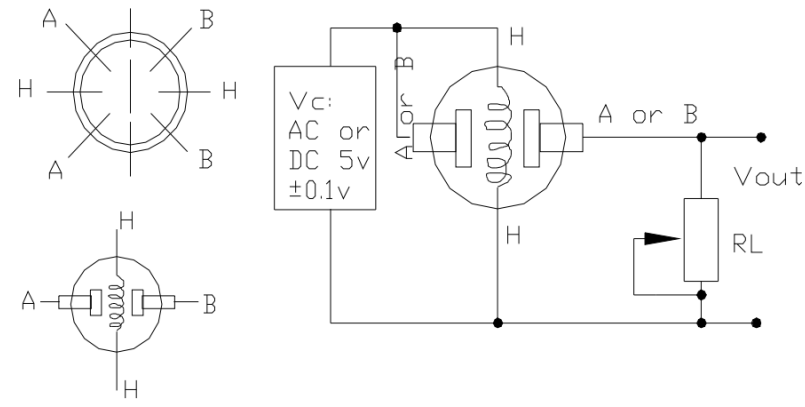

Fig. 7. Circuit schematic of smoke sensor.

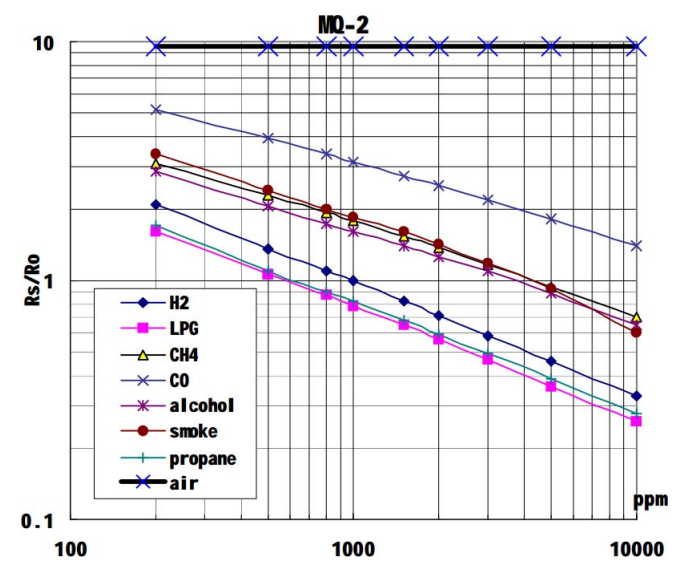

Fig. 8. Relationship between $R_{S} / R_{O}$ and gas concentrations for MQ2 sensor.

Finally, lux can be calculated as:

$$
\text { lux }=\frac{470.5437}{R[k \Omega]^{1.3132}}
$$

\section{F. Smoke Sensor}

To provide a measure of safety, an electrochemical gas sensor was connected to each sensor node to detect the presence of smoke. The MQ-2 sensor was selected due to its sensitivity, stability, long life and fast response. The circuit schematic in Fig. 7 shows how the sensor was connected to the MCU.

Pins A-H-A on the left hand-side were connected to $\mathrm{V}_{\mathrm{cc}}$; pin $\mathrm{H}$ on the right hand-side was connected to GND, and the B pins were connected to the MCU's ADC pins. Calibration graphs showing the relationship between $R_{S} / R_{O}$ and concentrations in parts per million (ppm) were used to detect the presence of smoke. It is noted that for this application, the detection of smoke is more important than the actual concentration in ppm so exact accuracy is not a concern. The clean air factor is observed to be around $9.83 \mathrm{k} \Omega$ from the calibration graph shown in Fig. 8.

\section{G. Control Node}

The control node serves two primary functions: it coordinates all other components of the system and manages intercomponent communication. It also analyses data received from

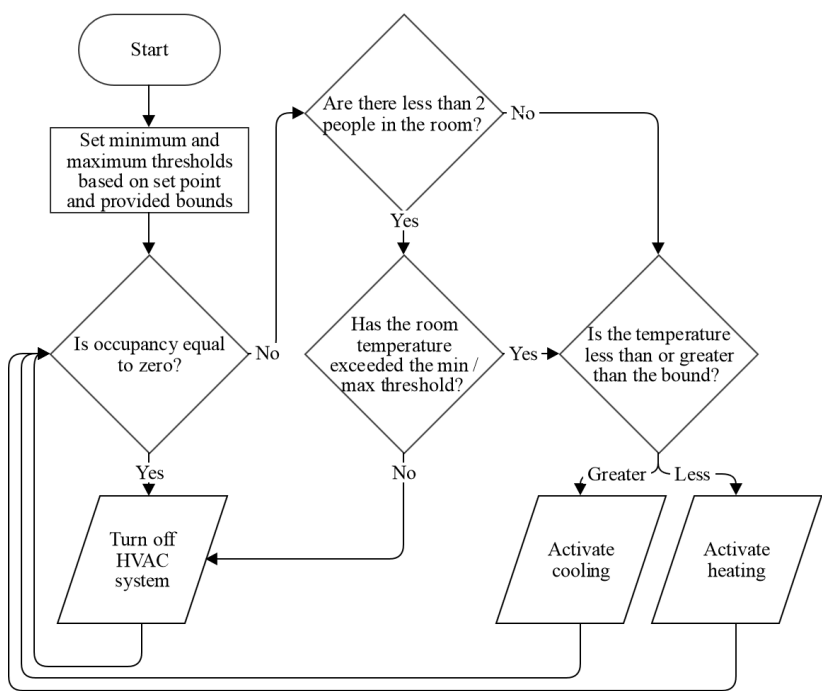

Fig. 9. Control algorithm running on control node.

the wireless sensor nodes and provides an adequate signal to the HVAC system.

The control node is equipped with Xbee and WiFi radios. The ESP8266 module is compatible with the IEEE $802.11 \mathrm{~b} / \mathrm{g} / \mathrm{n}$ standard and includes a 32-bit microcontroller to provide web application processing and hosts a webserver which can be accessed from any Hypertext Transfer Protocol (HTTP) client. Since the ESP8266 module handles all web processing and client connections, the PIC32MX220 MCU functions as a dedicated processor which executes the HVAC control algorithm.

\section{H. Control Algorithm}

The flow diagram shown in Fig. 9 describes the operation of the control algorithm which was implemented based on the simulation described in Section IV.B. It is executed on the control node's microcontroller.

The code enables the controller to activate heating or cooling based on the detected occupancy, which is based on the data received from the wireless sensors.

\section{Mobile Application}

The mobile application runs on an Android smartphone and allows the system's user to view the current status of the system and control it. The user interface is shown in Fig. 10.

This app allows the user to adjust the set point, view the history of each of the 5 sensors, and also alert the user in case of an emergency.

\section{Results and Discussion}

To evaluate the system, a number of tests on each sensor sub-system were conducted, according to experimental protocols described in this Section. For the sake of brevity, tests to evaluate the wireless connectivity of the system are omitted.

\section{A. Smoke}

The smoke sensor was configured to take readings every 2 seconds for 5 minutes. A mixture of $30 \mathrm{~g}$ potassium nitrate 


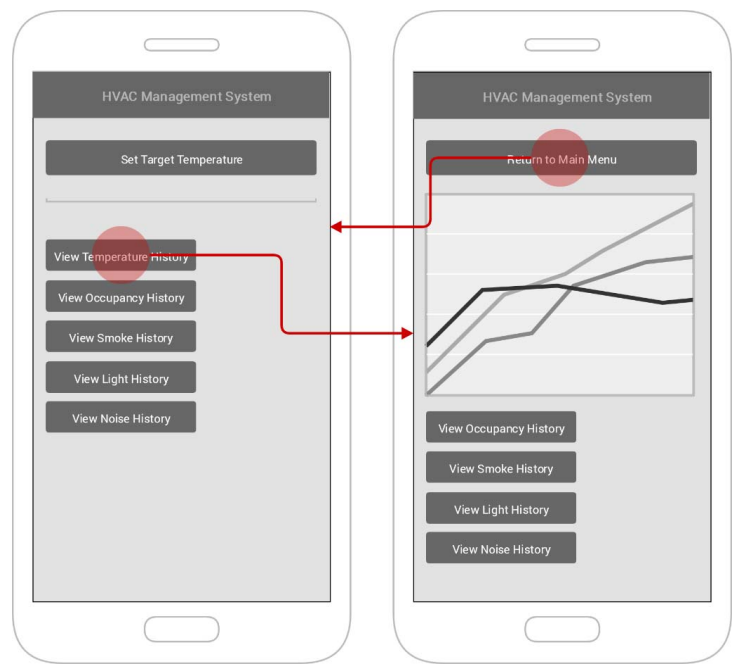

Fig. 10. User interface of mobile application.

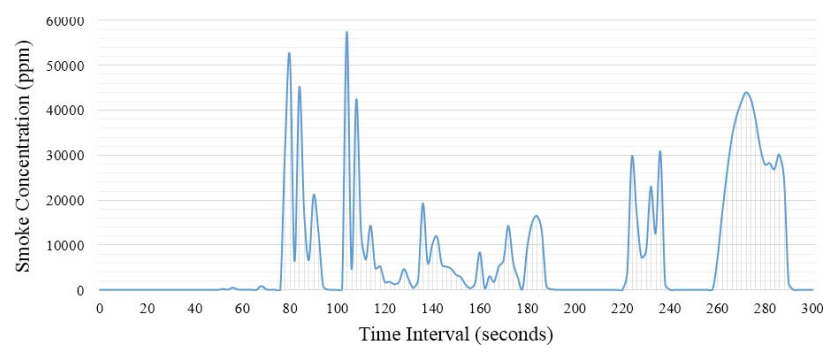

Fig. 11. Evaluation of smoke detector.

and $20 \mathrm{~g}$ table sugar was used to create smoke, which was then trapped in a container. Using a syringe as an applicator, smoke was administered directly to the sensor at varying rates. Fig. 11 shows the readings obtained with this procedure.

The 2 large spikes (at 75 and 105 seconds) are due to the entire $5 \mathrm{ml}$ capacity of the syringe being released instantly, whilst the much smaller spikes are due to lesser quantities of smoke being released at a time. Validating the accuracy of the smoke sensor was difficult since the reference was an estimation of the concentration of smoke in the syringe applicator. However, in this work, a binary indicator of the presence of smoke is more important than the actual measurement of smoke amount and it is shown that this sensor is adequate. This MQ-2 gas sensor allows each sensor node to accurately detect the presence of smoke in the room up to $5000 \mathrm{ppm}$. Above $5000 \mathrm{ppm}$, the readings become inaccurate but still provide a binary indication of the presence of smoke.

\section{B. Temperature}

Evaluation was performed over the range of $18{ }^{\circ} \mathrm{C}$ and $73{ }^{\circ} \mathrm{C}$. Samples for each temperature were collected at 2 second intervals with a total sample size of 366. Fig. 12 shows the results of this evaluation.

It is seen that temperature sensing was accurate to within $0.3{ }^{\circ} \mathrm{C}$ over the operating range of $18{ }^{\circ} \mathrm{C}$ to $60{ }^{\circ} \mathrm{C}$, and was always within specification. The temperature sensor was accurate to within $0.2^{\circ} \mathrm{C}$ when measuring temperatures within the range of approximately $10{ }^{\circ} \mathrm{C}$ to $60{ }^{\circ} \mathrm{C}$.

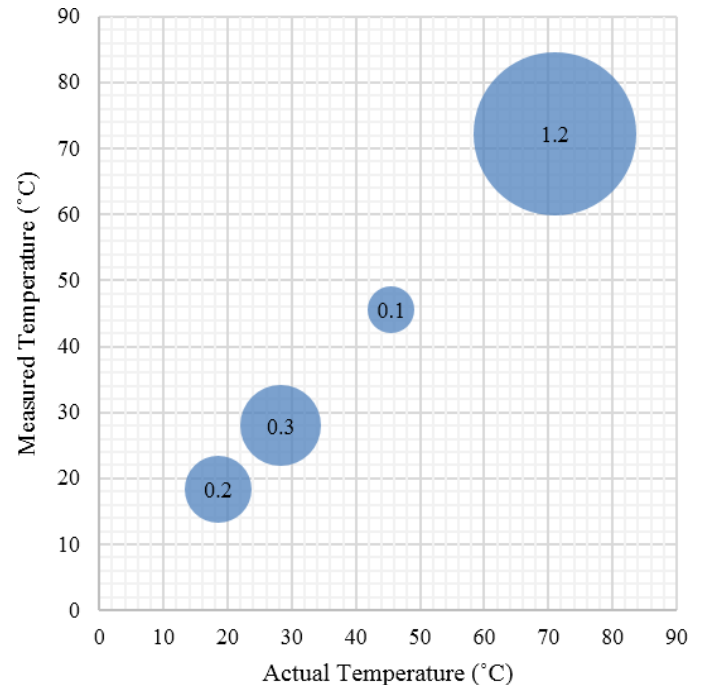

Fig. 12. Temperature sensing results.

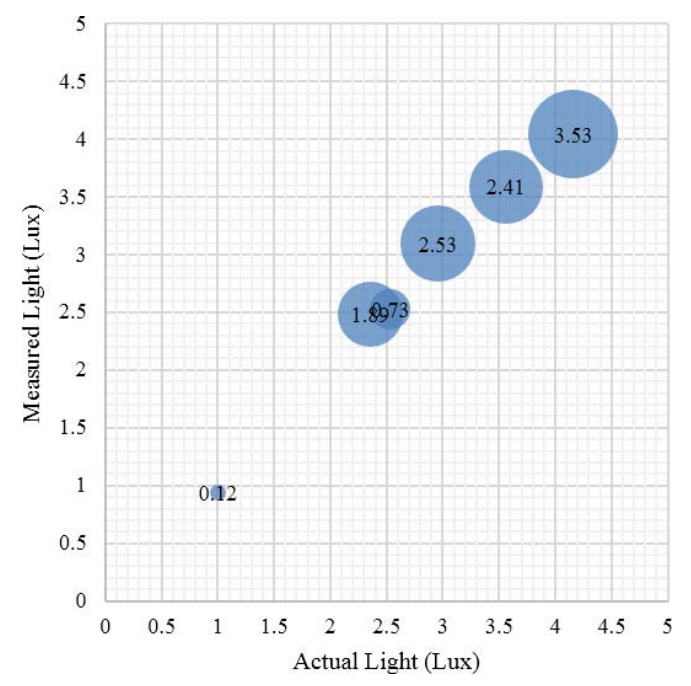

Fig. 13. Light sensing results.

\section{Light}

An evaluation was conducted using a variety of different levels of light exposure. A 600-lumen torch with a view angle of $90^{\circ}\left(45^{\circ}\right.$ on either side of the central beam) was shone directly at the light sensor from varying distances. Fig. 13 illustrates the measured light against known light at various intensities. Due to the large range (10 lux to 14490 lux) all values have been converted to $\log 10$.

The light sensor reported measurements varying with an error ranging from $15 \%$ to $27 \%$ when measuring light over the range of 10 lux and 400 lux. This can be possibly improved with the use of a photometer. Within a range of $10 \mathrm{~lx}$ to $3500 \mathrm{~lx}$, an error of between $1.33 \mathrm{~lx}$ and $338.13 \mathrm{~lx}$ was observed. Outside this range, the error rate increased dramatically to $3423 \mathrm{~lx}$ when attempting to measure a known value of $14490 \mathrm{~lx}$.

\section{Noise}

The first test was at the center of an office in order to find the environment's background noise. This was found to be 


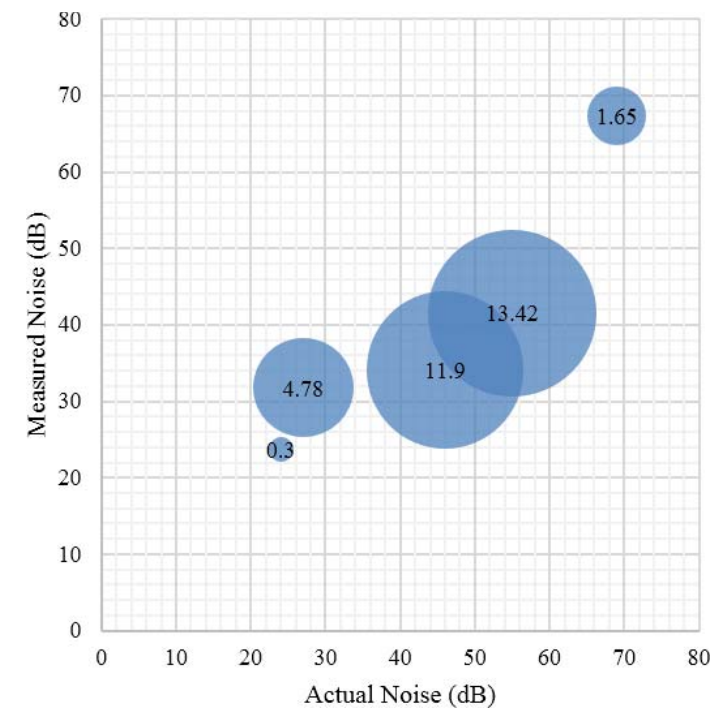

Fig. 14. Noise sensing results.

Table II. Summary of Trials Conducted on Simulink

\begin{tabular}{|l|l|l|}
\hline Trial & $\begin{array}{l}\text { Cost using on-off } \\
\text { controller }(\mathbf{R})\end{array}$ & $\begin{array}{l}\text { Cost using } \\
\text { improved } \\
\text { controller }\end{array}$ \\
\hline 1 & 159 & 106 \\
\hline 2 & 166 & 94 \\
\hline 3 & 164 & 93 \\
\hline 4 & 143 & 87 \\
\hline 5 & 172 & 104 \\
\hline Average & 160.8 & 96.8 \\
\hline
\end{tabular}

an average of $24 \mathrm{~dB}$. The actual noise levels were measured with a commercial off-the-shelf (COTS) sensor and the results between the 2 sensors were found to be similar, as shown in Fig. 14.

Deviation of the measurements from the actual values is depicted by the size of the circles around each point. The noise results in errors between $0.3 \mathrm{~dB}$ and $13.42 \mathrm{~dB}$. Practically, this value is equivalent to being able to hear someone breathe from around $1 \mathrm{~m}$ away, therefore it is considered a fairly good result.

\section{E. Occupancy Detection}

Occupancy detection is reliable provided one person enters the door at a time and that they walk at a reasonable pace. Running, walking very slowly, or walking in close proximity to other people can cause the system to miscount. More advanced approaches (like camera-based people counting) can be implemented to increase the reliability of the occupancy detection component of the system.

\section{F. Comparison of Bang-Bang Controlled HVAC and Improved Control Algorithm}

When simulating the efficiency of the on-off controller and the improved system, the simulations were each run for a total of 600 time steps which equates to 250 days. Table II shows the results of these experiments.

The percentage improvement (Imp) can be calculated as:

$$
\operatorname{Imp}(\%)=\frac{160.8-96.8}{160.8} * 100=39.8 \%
$$

Therefore, the percentage increase in efficiency is $39.8 \%$, showing that the improved algorithm outperforms the conventional bang-bang type of control.

\section{Conclusion}

In this paper, a proof-of-concept of a Web-based climate control system was presented. This system relies on sensor data from multiple sensors to detect temperature, noise, light, smoke and occupancy levels. An improved HVAC control algorithm that is more advanced than the conventional on-off scheme was simulated in Simulink, and shown to provide an improvement of $39.8 \%$ in terms of energy efficiency. Experiments showed that the selected sensors provide data that is reliable and within requirement specifications.

For future work, the system developed in this work will be integrated with an HVAC system to evaluate its performance in a realistic environment. Additionally, the history of the system could be recorded for longer time periods. Consideration should be given to a full Apache, PHP and MySQL stack which can easily store large amounts of information. Alternatively, EEPROM could be used for permanent data storage on the control node.

\section{References}

[1] Y. Tachwali, H. Refai, and J. E. Fagan, "Minimizing HVAC energy consumption using a wireless sensor network," in Proc. 33rd Annu. Conf. IEEE Ind. Electron. Soc. (IECON), Taipei, Taiwan, Nov. 2007, pp. 439-444.

[2] R. Fisher, L. Ledwaba, G. P. Hancke, and C. Kruger, "Open hardware: A role to play in wireless sensor networks?" Sensors, vol. 15, no. 3, pp. 6818-6844, 2015.

[3] J. Brooks et al., "Experimental evaluation of occupancy-based energyefficient climate control of VAV terminal units," Sci. Technol. Built Environ., vol. 21, no. 4, pp. 5680-5685, 2015.

[4] The SmartME Controller: Its Brilliance and Simplicity, accessed on Apr. 5, 2016. [Online]. Available: http://www.mitsubishipro.com/newcontrollers

[5] Honeywell Building Controls-Sensors, accessed on Apr. 5, 2016 [Online]. Available: https://buildingcontrols.honeywell.com/subcategory/ Sensors

[6] Honeywell Building Controls-HVAC Controllers, accessed on Apr. 5, 2016. [Online]. Available: https:buildingcontrols.honeywell.com/ HVAC-Controllers

[7] H. Y. Tung et al., "The generic design of a high-traffic advanced metering infrastructure using ZigBee," IEEE Trans. Ind. Informat., vol. 10 , no. 1, pp. 836-844, Feb. 2014.

[8] Y. Wang, H. Feng, and X. Xi, "Sense, model and identify the load signatures of HVAC systems in metro stations," in Proc. IEEE PES General Meeting Conf. Expo., Jul. 2014, pp. 1-5.

[9] S. Purdon, B. Kusy, R. Jurdak, and G. Challen, "Model-free HVAC control using occupant feedback," in Proc. IEEE 38th Conf. Local Comput. Netw. Workshops (LCN Workshops), Oct. 2013, pp. 84-92.

[10] J. Liang and R. Du, "Thermal comfort control based on neural network for HVAC application," in Proc. IEEE Conf. Control Appl., Toronto, ON, Canada, Aug. 2005, pp. 819-824.

[11] A. Rahmati, F. Rashidi, and M. Rashidi, "A hybrid fuzzy logic and PID controller for control of nonlinear HVAC systems," in Proc. IEEE Int. Conf. Syst., Man, Cybern., Oct. 2003, pp. 2249-2254. 
[12] J. Fütterer, P. J. E. Kraus, M. Schmidt, and D. Müller, "Demonstration of an easy-to-apply, automated control tuning method for typical PID control loops in building energy systems," in Proc. 10th Conf. Ind. Electron. Appl. (ICIEA), Jun. 2015, pp. 2069-2075.

[13] J. Liu, W.-J. Cai, and G.-Q. Zhang, "Design and application of handheld auto-tuning PID instrument used in HVAC," in Proc. 4th IEEE Conf. Ind. Electron. Appl., May 2009, pp. 1695-1698.

[14] Your Complete Source for Building Automation Sensors, accessed on Apr. 5, 2016. [Online]. Available: http://www.automatedbuildings.com/ news/nov12/articles/enlighted/121026014101enlighted.html

[15] S. M. Saad, A. R. M. Saad, A. M. Y. Kamarudin, A. Zakaria, and A. Y. M. Shakaff, "Indoor air quality monitoring system using wireless sensor network (WSN) with Web interface," in Proc. Int. Conf. Elect., Electron. Syst. Eng., 2013, pp. 60-64.

[16] D. Brunelli, I. Minakov, R. Passerone, and M. Rossi, "Smart monitoring for sustainable and energy-efficient buildings: A case study," in Proc. IEEE Workshop Environ., Energy Struct. Monitor. Syst. (EESMS), Jul. 2015, pp. 186-191.

[17] D. Zhu, S. Beepy, M. Tudor, N. White, and N. Harris, "Novel miniature airflow energy harvester for wireless sensing applications in buildings," IEEE Sensors J., vol. 13, no. 2, pp. 691-700, Feb. 2013

[18] Y. Wang, M. Nishikawa, R. Maeda, M. Fukunage, and K. Watanabe, "A smart thermal environment monitor based on IEEE 1451.2 standard for global networking," IEEE Trans. Instrum. Meas., vol. 54, no. 3, pp. 1321-1326, Jun. 2005.

[19] A. Kumar and G. P. Hancke, "Energy efficient environment monitoring system based on the IEEE 802.15.4 standard for low cost requirements," IEEE Sensors J., vol. 14, no. 8, pp. 2557-2566, Aug. 2014.

[20] A. Kumar and G. P. Hancke, "An energy-efficient smart comfort sensing system based on the IEEE 1451 standard for green buildings," IEEE Sensors J., vol. 14, no. 12, pp. 4245-4252, Dec. 2014.

[21] S. Atthajariyakul and T. Leephakpreeda, "Real-time determination of optimal indoor-air condition for thermal comfort, air quality and efficient energy usage," Energy Buildings, vol. 36, no. 7, pp. 720-733, 2004.

[22] C. Peng, K. Qian, and C. Wang, "Design and application of a VOC-monitoring system based on a ZigBee wireless sensor network," IEEE Sensors J., vol. 15, no. 4, pp. 2255-2268, Apr. 2015.

[23] B. Silva, R. M. Fisher, A. Kumar, and G. P. Hancke, "Experimental link quality characterization of wireless sensor networks for underground monitoring," IEEE Trans. Ind. Informat., vol. 11, no. 5, pp. 1099-1110, Oct. 2015

[24] A. Nag and S. C. Mukhopadhyay, "Occupancy detection at smart home using real-time dynamic thresholding of flexiforce sensor," IEEE Sensors J., vol. 15, no. 8, pp. 4457-4463, Aug. 2015.

[25] K. Akkaya, I. Guvenc, R. Aygun, N. Pala, and A. Kadri, "IoT-based occupancy monitoring techniques for energy-efficient smart buildings," in Proc. IEEE Wireless Commun. Netw. Conf. Workshops (WCNCW), New Orleans, LA, USA, Mar. 2015, pp. 58-63.

[26] F. Wahl, M. Milenkovic, and O. Amft, "A distributed PIR-based approach for estimating people count in office environments," in Proc. IEEE 15th Int. Conf. Comput. Sci. Eng. (CSE), Nicosia, Cyprus, Dec. 2012, pp. 640-647.

[27] N. Tang, Y.-Y. Lin, M.-F. Weng, and H.-Y. M. Liao, "Cross-camera knowledge transfer for multiview people counting," IEEE Trans. Image Process., vol. 24, no. 1, pp. 80-93, Jan. 2015.

[28] H. Lv et al., "Multi-target human sensing via UWB bio-radar based on multiple antennas," in Proc. IEEE Region 10 Conf. (TENCON), Xi'an, China, Oct. 2013, pp. 1-4.

[29] S4ECoB Project: Sounds for Energy Control of Buildings, accessed on Apr. 5, 2016. [Online]. Available: http://www.s4ecob.eu/

[30] G. Deak, K. Curran, J. Condell, and D. Deak, "Detection of multioccupancy using device-free passive localisation," IET Wireless Sensor Syst., vol. 4, no. 3, pp. 130-137, Sep. 2014.

[31] S. Savazzi, V. Rampa, F. Vicentini, and M. Giussani, "Device-free human sensing and localization in collaborative human-robot workspaces: A case study," IEEE Sensors J., vol. 16, no. 5, pp. 1253-1264, Mar. 2016.

[32] Z. Yang, N. Li, B. Becerik-Gerber, and M. Orosz, "A multi-sensor based occupancy estimation model for supporting demand driven HVAC operations," in Proc. Symp. Simulation Archit. Urban Design, 2012, Art. no. 2.
[33] M. Wang, X. Wang, G. Zhang, and C. Li, "Occupancy detection based on spiking neural networks for green building automation systems," in Proc. 11th World Congr. Intell. Control Autom. (WCICA), Shenyang, China, 2014, pp. 2681-2686.

[34] J. L. G. Ortega, L. Han, N. Whittacker, and N. Bowring, "A machinelearning based approach to model user occupancy and activity patterns for energy saving in buildings," in Proc. Sci. Inf. Conf. (SAI), London, U.K., 2015, pp. 474-482.

[35] B. Dong, K. P. Lam, and C. Neuman, "Integrated building control based on occupant behavior pattern detection and local weather forecasting," in Proc. 12th Int. IBPSA Conf., 2011, pp. 193-200.

[36] Z. Han, R. X. Gao, and Z. Fan, "Occupancy and indoor environment quality sensing for smart buildings," in Proc. IEEE Int. Instrum. Meas. Technol. Conf. (I2MTC), May 2012, pp. 882-887.

[37] A. Akbar, M. Nati, F. Carrez, and K. Moessner, "Contextual occupancy detection for smart office by pattern recognition of electricity consumption data," in Proc. IEEE Int. Conf. Commun. (ICC), London, U.K., Jun. 2015, pp. 561-566.

[38] (2015). Buildings Energy Data Book, accessed on Jul. 26, 2015. [Online]. Available: http://buildingsdatabook.eren.doe.gov/

[39] Z. Yang and B. Becerik-Gerber, "Coupling occupancy information with HVAC energy simulation: A systematic review of simulation programs," in Proc. Winter Simulation Conf. (WSC), Dec. 2014, pp. 3212-3223.

[40] K. Liu and Z. Tian, "Simulation and factor experiment analysis for cooling and heating load of terminal based on TRNSYS," in Proc. 2nd Int. Conf. Mech. Autom. Control Eng. (MACE), Jul. 2011, pp. 3422-3425.

[41] J. T. Jaber-López, I. Valencia-Salazar, E. Peñalvo-López, C. Álvarez-Bel, D. C. Gamboa-Olivares, and E. Merino-Hernández, "Analysis comparison of energy efficiency: CALENER vs TRNSYS," in Proc. 3rd Int. Youth Conf. Energetics (IYCE), Jul. 2011, pp. 1-5.

[42] G. Goddard, J. Klose, and S. Backhaus, "Model development and identification for fast demand response in commercial HVAC systems," IEEE Trans. Smart Grid, vol. 5, no. 4, pp. 2084-2092, Jul. 2014.

[43] M. Turunen, O. Toyinbo, T. Putus, A. Nevalainen, R. Shaughnessy, and U. Haverinen-Shaughnessy, "Indoor environmental quality in school buildings, and the health and wellbeing of students," Int. J. Hygiene Environ. Health, vol. 217, no. 7, pp. 733-739, 2014.

[44] T. Itani, N. Ghaddar, and K. Ghali, "Strategies for reducing energy consumption in existing office buildings," Int. J. Sustain. Energy, vol. 32, no. 4, pp. 259-275, 2013

[45] O. Guerra-Santin and L. Itard, "Occupants' behaviour: Determinants and effects on residential heating consumption," Building Res. Inf., vol. 38, no. 3, pp. 318-338, 2010

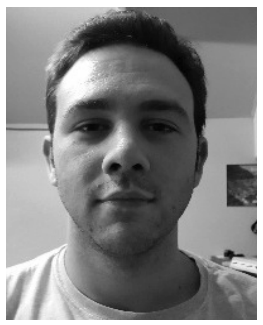

Timothy W. Foster received the B.Eng. degree from the University of Pretoria, South Africa, in 2015 $\mathrm{He}$ is currently pursuing his career in the industry.

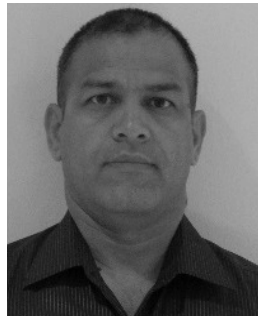

Deep Vardhan Bhatt received the B.Eng. degree from ATBU, Nigeria, and the M.Eng. degree from the University of Pretoria, South Africa, in 2011. He is a Lecturer with the Department of Electrical, Electronic and Computer Engineering. He is part of the Advanced Sensor Network Research Group. His current interests are in the free space optical communication, Internet of Everything, and sensor routing for optimum power utilization. 


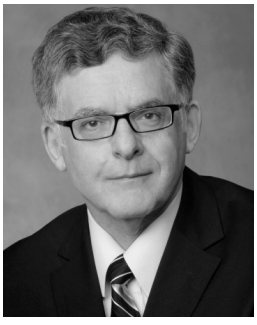

Gerhard P. Hancke (F'16) received the B.Sc., B.Eng., and M.Eng. degrees from the University of Stellenbosch, South Africa, and the D.Eng. degree from the University of Pretoria, South Africa, in 1983. He is with the University of Pretoria, where he is currently a Professor and Coordinator of the Computer Engineering Program and Head of the Advanced Sensor Networks (ASN) Research Group, which has links with the Meraka Institute, Council for Scientific and Industrial Research. He is recognized internationally as a Pioneer and Leading Scholar in advanced sensor networks research, especially aimed at industrial applications.

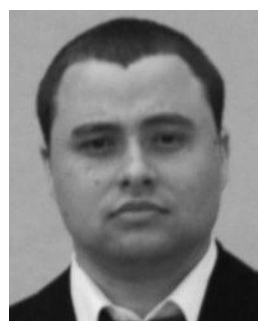

Bruno Silva (S'14) received the B.Eng., B.Eng. (Hons.), and M.Eng. degrees from the University of Pretoria, Pretoria, South Africa, in 2011, 2012, and 2015, respectively, all in computer engineering.

$\mathrm{He}$ was a Visiting Researcher with the $\mathrm{ABB}$ Corporate Research Centre, Västerås, Sweden, from 2013 to 2014, where he was involved in research on ultra-wideband based localization. His research interests include wireless sensor networks, digital signal processing, and mobile computing. 